\title{
Maximizing, choice freedom, and duration judgments in choice making
}

\author{
Nicky Rogge
}

Faculty of Economics and Business (ECON), Katholieke Universiteit Leuven, Warmoesberg 26, B-1000 Brussels, Belgium

\section{Correspondence}

Nicky Rogge, Faculty of Economics and Business (ECON), Katholieke Universiteit Leuven (Belgium), Warmoesberg 26, B-1000 Brussels, Belgium.

Email: nicky.rogge@kuleuven.be

\begin{abstract}
This paper examines (a) whether people are less accurate in judging choice time as choice tasks involve more choice options, more choice information, or a combination of both and (b) whether people with a higher tendency to look for the best option in choice making (maximizers) have less accurate duration judgments of choice time as compared to people who are more easily settling for a choice outcome that is satisfactory (satisficers). A multilevel analysis is used to explore the relationships of interest using data collected through a series of choice tasks. In general, the results suggest that people seem to misjudge time durations when making choices. Moreover, empirical evidence demonstrates that people with an outspoken tendency to maximize in choice making do not differ significantly in estimating choice time accurately as compared to people who experience almost no need to maximize.
\end{abstract}

KEYWORDS

choice complexity, choice tasks, decision or choice making, duration judgment, maximizing

\section{1 | INTRODUCTION}

The conventional wisdom in Western societies suggests that more choice freedom and flexibility are, almost by definition, better for people. This wisdom builds on the belief that the availability of more choice options and choice information benefits people as it (a) increases the prospects of each individual finding products, services, experiences, and so on that better match his or her own preferences so as to yield a high utility; (b) gives people more opportunities to keep options open for eventual future changes of tastes and preferences (e.g., Kahneman \& Snell, 1992); and (c) makes people feel more in control of their lives (Ryan \& Deci, 2000). In spite of this widely held belief, researchers in the psychology, behavioral economics, and consumer choice literature have increasingly questioned this popular notion of more choice being better (Chernev, 2003; lyengar \& Lepper, 2000; lyengar, Wells, \& Schwartz, 2006; Schwartz, 2004). Though most of them recognize that more choice can be beneficial to people, they warn for the other side of the coin, more choice freedom hampering people in their attempt to make good choices. For instance, in his recent bestseller The Paradox of Choice: Why More Is Less, Barry Schwartz (2004, p. 3) remarks that "the fact that some choice is good doesn't necessarily mean that more choice is better." In other words, whereas in some situations or to some extent, more choice freedom benefits individuals, in other cases or beyond certain levels (particularly when choice becomes abundant in every aspect of life), more choice freedom becomes a harness to the majority of the people, thereby causing, among other things, higher choice-related costs and potentially lower quality choice outcomes.

A broad literature has explored how choice freedom relates to the quality of the choices made by individuals and the amount of resources spent in choice making (e.g., Bettman, Luce, \& Payne, 1998). The important findings of these studies are that as choice tasks are more complex due to more choice options, more choice information, or a combination of both, emotional costs for the choice maker are higher (e.g., due to difficulties in trading off the strengths and weaknesses of each choice option; lyengar \& Lepper, 2000), overall choice consistency is lower (DeShazo \& Fermo, 2002), the magnitude of suboptimal mistakes is higher (De Palma, Myers, \& Papageorgiou, 1994), the likelihood of making good choices is lower (Botti \& McGill, 2006; Chernev, 2003; White \& Hoffrage, 2009), discomfort or paralysis experienced by individuals is higher (lyengar \& Lepper, 2000; Sanchis, Rey, \& Álvarez, 2014), and choice effectiveness is lower (Keller \& 
Staelin, 1987). ${ }^{1}$ Sanchis et al. (2014) demonstrated how increasing the number of options of a given product may lower consumer welfare. There is also a large literature showing a positive relation between the size of the choice assortment and choice time; that is, choice makers spend more time in choice making as more choice options, more choice information, or both are available (Arunachalam, Henneberry, Lusk, \& Norwood, 2009; Botti \& lyengar, 2006; lyengar \& Lepper, 2000; Schwartz, 2004). ${ }^{2}$ Several intuitions have been proposed for explaining this positive relationship. One explanation is that complex choice situations complicate and obscure the choice-making task for people, which makes the task more difficult and hence timeconsuming to solve (Chernev, 2003; lyengar \& Lepper, 2000 and Schwartz et al., 2002). Somewhat related to this, Fasolo, Carmeci, and Misuraca (2009) and Fasolo, McClelland, and Todd (2007) argued that the presence of more choice options and/or choice information makes it more likely that people will experience choice conflicts. This makes it more difficult and time-consuming to find an alternative in the choice set that is eligible for selection.

Several studies argued and demonstrated that choice freedom may be more salient for some types of individuals than for others, depending on their choice-making strategy or style, goal in choice making, personality traits, cognitive abilities, and more (Cheek \& Schwartz, 2016). One such individual characteristic that has been frequently studied in the context of choice making is whether the individual adopts a maximizing or satisficing strategy in choice making. The concepts of maximizer and satisficer date back to the work of Herbert Simon $(1955,1956)$ who introduced these concepts as more realistic representations of human beings as choice makers (see Dalal, Diab, Zhu, \& Hwang, 2015, for a more elaborate discussion). Maximizers are somewhat imperfect versions of the homo economicus as postulated by classical economic theory (Von Neumann \& Morgenstern, 1953), in that they aim for the best yet exhibit cognitive limitations. Satisficers, on the other hand, are individuals who in a lot of situations look for satisfactory outcomes (i.e., outcomes which are good enough, however, not necessarily the best). Relative to satisficers, maximizers have been found to be more hopeless in choice making (Bruine de Bruin, Dombrovski, Parker, \& Szanto, 2016), more prone to regret (Besharat, Ladik, \& Carrillat, 2014; Parker, Bruine de Bruin, \& Fischhoff, 2007; Schwartz et al., 2002), and less committing to the choice (Sparks, Ehrlinger, \& Eibach, 2012). Parker et al. (2007) found that people with a higher tendency to maximize are more likely to apply maladaptive styles for choice making and more avoiding of choice making. Other studies like those of Dijksterhuis, Bos, Nordgren, and Van Baaren (2006) and Chowdhury, Ratneshwar, and Mohanty (2009) demonstrated that as the number of choice options increases, maximizers become increasingly overwhelmed by a feeling of losing grip. Arunachalam et al. (2009) found that maximizers are more prone to

${ }^{1}$ As to the link between the number of choice options and the quality of the choice outcome, it is important to note that the finding of a negative association is contested by other studies that found that the link is rather weak (Scheibehenne, Greifeneder, \& Todd, 2010), positive (Boatwright \& Nunes, 2001), or curvilinear (Shah \& Wolford, 2007).

${ }^{2}$ DeShazo and Fermo (2002) and Haynes (2009) argued that the relation between the number of options in the choice set and the amount of choice time could be an inverted-U shape. suffer from the excessive-choice effect than their counterparts who are less inclined to do so. Similarly, Fasolo et al. (2009) discussed that maximizers are more likely to suffer from choice overload as they focus more on how options compare relative to each other rather than on the absolute performances of options. People with lower maximizing tendencies, on the other hand, in their quest for a merely satisfactory option, evaluate options more based on their absolute performance values. As to the relationship between maximizing and subjective well-being, Kokkoris (2016) demonstrated that whereas maximizing has been found to relate negatively to hedonic well-being (Purvis, Howell, \& lyer, 2011), it may very well be positively associated with eudaimonic well-being (i.e., well-being derived from the development of one's best potential and their application in the fulfillment of selfconcordant goals). As to the resources spent in choice making, Schwartz et al. (2002) demonstrated that maximizers and satisficers differ in their motivation to invest time, cognitive effort, and other types of resources in the choice-making process. Nenkov, Morrin, Ward, Schwartz, and Hulland (2008) and Scheibehenne et al. (2010) compared the time needed for maximizers and satisficers to perform similar choice tasks and found that generally maximizers require more time in order to come to a final choice. Dar-Nimrod, Rawn, Lehman, and Schwartz (2009) demonstrated that people who have a higher motivation to look for the best choice option encounter more search costs (i.e., time and costs spent on search) relative to their counterparts. lyengar et al. (2006) found that maximizers, because of their higher tendency to explore more options, are typically incurring higher investment cost.

The present paper is part of a larger research project that explores the consequences of presenting more choice options and choice information to people on actual choice time and the people's perception of choice time. A first paper (Rogge, 2016) scrutinized the link between choice freedom and choice time. In particular, in that paper, it was explored how the availability of more choice options, more choice information (as measured by the number of attributes with choice information), or a combination of both relates to the amount of time spent in performing choice tasks. The empirical results collected from a series of choice tasks that were conducted with 309 undergraduate students indicated that people generally spend increasingly more time in choice making as choice tasks involve more choice options, choice information, or a combination of both. The conclusion of the paper was that increasing the amount of choice information or expanding the number of choice options beyond a certain threshold results in more time being spent in choice making. A special focus in this paper was on the difference in choice time between maximizers and satisficers. Whereas most previous studies demonstrated that maximizers are more likely to spend more time in choice making as compared to satisficers, results in that first paper did not show any significant differences in choice time between both types of choice makers. As a possible explanation, the study indicated the fact that the analysis controlled for several features of the choice task and respondent characteristics (e.g., gender), which was typically not or only partially done in most other studies.

The present paper presents the second part of the research project. The focus in this paper is on the link between choice freedom and people's perception of choice time. The paper makes a 
contribution to the maximization literature in several ways. First, the paper examines whether (and, if so, how) the available number of choice options and/or choice information relates to the accurateness of people's duration judgments of choice time. The focus is on time durations of several minutes. This deviates from previous studies that mostly focused on relatively short time spans ranging from a couple of seconds to a minute (Brown \& Boltz, 2002; Brown \& West, 1990; Burnside, 1971; Woehrle \& Magliano, 2012). In fact, whereas many of the day-by-day decisions such as going to the bakery or to the supermarket are done in some cases almost instantaneously or at least without spending several minutes deciding on what product to buy, decisions on things with a higher impact on monetary, social, or other contexts (such as choosing a job, choosing a working partner, choosing a holiday destination, buying a car, or buying a house) typically require longer time. Second, this study examines people's duration judgments of time in choice-making settings, whereas previous studies typically investigated people's perceptions of time durations in other contexts (e.g., solving analogies, target stimulus, melodies, perceptual-motor tasks, computation tasks, and memorizing information; Block, Hancock, \& Zakay, 2010; Brown, 1997; Brown \& Boltz, 2002; Brown \& West, 1990; Carmeci, Misuraca, \& Cardaci, 2009; Mantel \& Kellaris, 2003; Woehrle \& Magliano, 2012). Third, and somewhat related to the second point, contrary to previous studies (e.g., Fasolo et al., 2009), we scrutinize the accurateness of people's duration judgments of choice time and the link with the amount of choice information across a variety of choice tasks. In particular, choice settings that involve five different types of choice stimuli are designed: booking a holiday hotel, booking a restaurant, selecting a partner for a work project, buying a smartphone, and choosing a job. The idea is that studying the same sample of individual across this series of choice tasks enables investigating the relationships of interest more profoundly. The choice for the five particular choice stimuli was driven by the aim to select choice stimuli that (also in real-life situations) require the average individual to spend considerable cognitive effort, deliberation, and time. Fourth, the paper proposes a novel measure of duration judgment to assess the accurateness of people's duration judgments, which differs from previously used duration judgment ratios (Block et al., 2010; Misuraca \& Teuscher, 2013; Woehrle \& Magliano, 2012). In particular, to allow for a better distinction between accurate and inaccurate duration judgments of choice time in a multilevel modeling (MLM) analysis, a normalized version of the duration judgment ratio is proposed. Fifth, an important contribution of this study is that it aims at providing a better understanding of how people's tendency to maximize in choice making relates to the precision of their judgment on choice time. In sum, the study aims at addressing the following two key research questions: (a) does the accurateness of people's perceptions of choice time alter as choice tasks involve more choice options and/or information and (b) does people's tendency to maximize and, hence, look for the best possible outcome in choice making relate to the precision of their duration judgments of choice time? An important motivation for answering these questions is the findings of previous studies that consumers are sensitive to time costs and that shopping behavior is strongly influenced by perceived time spent shopping (e.g., Ackerman \& Gross, 2003; Mantel \& Kellaris, 2003). Moreover, as demonstrated by Jessup, Veinott, Todd, and Busemeyer (2009), people take into account the time it takes them to make a choice in the sense that they use a personally and implicitly determined time limit for making choices. The key notion of Jessup et al.'s (2009) "time-out" hypothesis is that people exit the choice process (Jessup et al. refer to this as "leaving the story empty handed") when they feel they have reached this time limit. All of this makes people's perception of choice time a crucial element. To the best of my knowledge, I cannot recall a maximization study that explores people's perception of choice time while varying the number of choice options and the choice task. Therefore, I believe that the present paper extends the maximization literature.

Data were collected from a series of choice tasks that were conducted with 309 undergraduate students enrolled in the first year of the Business Administration program at the University of Leuven (Belgium). An MLM approach was employed to account for the hierarchical nature of the choice data, with participants completing several choice tasks (two-level MLM).

The paper is organized as follows. The next section describes the literature on time duration judgments and maximizing tendency in choice and decision making. On the basis of the findings of previous research, hypotheses are formulated. The ensuing section briefly describes that data set and the methodology to test the hypotheses. Then the results are presented and discussed. The concluding section summarizes the most important findings and formulates some implications. This section also discusses some limitations to the present study and several interesting areas for future research.

\section{2 | LITERATURE}

The psychology research literature discusses several theoretical models about human duration judgments (see, e.g., Block, 2003, for a review). Several of these theoretical models put forward the notion that the accuracy of people's duration judgments relates to what occurred during the time period. In particular, several models hypothesize that more complex tasks make it more difficult for people to keep track of time as fewer attentional resources are available for temporal information processing (Block \& Zakay, 2008; Zakay \& Block, 2004). One such a theory is the attentional resource theory as discussed by Kahneman (1973). Basically, this theory states that in settings in which people have to perform multiple tasks, an increase in the cognitive load of one of the tasks decreases the performance on the second task. In the context of choice making, this would imply that as choice tasks are more complex due to the presence of more choice options and/or more choice information, it becomes more difficult for the choice maker to keep track of time spent in performing the choice task. As a result, people will generally judge choice time less accurately. The intuition for this theory is largely based on the notion that humans have only limited attentional capacity (Block \& Zakay, 1997). Other theoretical frameworks advocate somewhat similar ideas. For instance, the resource-matching theory (see, e.g., Mantel \& Kellaris, 2003) states that the accurateness of people's durations judgments depends on the match between the available and required cognitive resources. According to this theory, the better the match between what is needed and what is available in terms of cognitive resources to perform a choice task properly, the more accurate the duration judgments. 
Another theoretical framework is the memory change model (Block \& Reed, 1978), which argues that duration judgments are related to the amount of stored and retrieved information. More in particular, this model hypothesizes that the average person judges time durations as longer in novel and/or more complex task environments as such environments typically require a person to put more effort in information encoding and processing. In the context of choice making, this would imply that people will on average judge choice time less accurately in novel and/or complex choice task environments. Other examples of models discussed in the literature include, among others, the contextual change model (e.g., Block, 2003; Block \& Reed, 1978), the attentional gate model (Zakay \& Block, 1997), and attention-based model (Thomas \& Weaver, 1975). As to the empirical evidence, Fasolo, Hertwig, Huber, and Ludwig (2009) and Brown and West (1990) demonstrated that tasks necessitating high mental workloads, such as selecting options from large and/or complex option sets, usually result in less accurate duration judgments.

In the maximization literature, only few studies attempted to tackle the question of how people's accurateness of duration judgments relates to amount of choice freedom (the number of choice options) and/or their tendency to maximize in decision and choice making. Notable exceptions include Fasolo et al. (2009), Besharat et al. (2014), and Misuraca and Teuscher (2013). Without controlling for the maximizing tendency of people, Fasolo et al. demonstrated that the perception of time spent making a choice is affected by the number of choice options available. More in particular, it was found that people underestimate choice time when being confronted with a large number of choice options and overestimate choice time when facing a small number of options in the choice set.

As to the difference between maximizers and satisficers in perceiving choice time accurately, Fasolo et al. (2009) advocated that people with a higher tendency to maximize are more likely to have an inaccurate perception of the time spent choosing. The reasoning is that maximizers experience choice tasks to be more complex and cognitive demanding due to the fact that they are typically engaging in an in-depth information search (Schwartz et al., 2002) and focusing more on how choice options compare to each other rather than on their absolute attribute values (Fasolo et al., 2009). Somewhat akin, Misuraca and Teuscher (2013) hypothesized that people with a higher tendency to maximize are more likely misjudge choice time as they spend more cognitive resources on the encoding and processing of choice information and less cognitive resources on keeping track of choice time. According to Besharat et al. (2014), as choice sets involve more choice options and/or more choice information, maximizers are more apt to experience a higher cognitive workload and, for that reason, suffer from tunnel vision in choice making, which causes them to be less aware of the factor time. The limited empirical evidence (mostly collected by Misuraca \& Teuscher, 2013) largely confirms these hypotheses. In particular, the outcomes of the choice tasks demonstrated that maximizers and satisficers perceived time durations differently during decision- and choice-making tasks (however, not during nondecisional tasks). Maximizers were found to persistently misjudge choice time irrespective of the specific requirements of the choice task. Satisficers, on the other hand, misjudged choice time only for the more challenging choice tasks. However, as Misuraca and Teuscher explored this link only across a limited number of task settings, more research is needed to validate this finding of maximizers being more likely to misjudge time spent choosing, compared to their satisficing counterparts. Nevertheless, on the basis of the limited empirical evidence and the intuitive reasoning derived from the theoretical models advocated in the psychology literature, the following hypothesis is advanced.

H1 Individuals with a higher tendency to maximize in choice making are less accurate in judging time spent choosing than individuals with lower tendencies to maximize, all else being equal.

Combining the theory and the empirical evidence as discussed in the two previous subsections, there are reasons to believe that the aforementioned factors, that is, the variables measuring the cognitive load of the choice task and the maximizing tendency of the participant, interact. As noted above, Fasolo et al. (2009) demonstrated that people with a higher tendency to maximize in their search for the best outcome focus more on how options compare to each other rather than on the absolute performances of options. People with lower maximizing tendencies, on the other hand, tend to evaluate choice options more based on their absolute performance values. Fasolo et al. discussed that the stronger focus of maximizers on how choice options compare to each other makes them more prone to suffer from choice overload. Obviously, due to this different focus in evaluating the performances of choice options, as the cognitive load of the choice task is higher due to a higher number of choice options and/or more choice information, the difference in choice overload experienced by maximizers and satisficers is likely to be larger, all else being equal. Straightforwardly, as several theoretical models about human duration judgments hypothesize a negative relationship between choice overload and accuracy in keeping track of the time duration, the following hypothesis is therefore tested.

H2 Less (More) choice options and choice-relevant information will attenuate (appreciate) the difference in the accuracy of duration judgment of choice time spent between maximizers and satisficers, all else being equal.

To conclude this literature section, it is important to note that some studies (e.g., Block, Hancock, \& Zakay, 2000; Coelho et al., 2004; Pande \& Pati, 2010) demonstrated that other personal characteristics may also relate to the accuracy of the people's perceptions of time durations. Examples of personal characteristics that have been demonstrated to be related to the accuracy in judging time durations are age, gender, emotional involvement, memory capacity, and so on. Wearden (2005) demonstrated that people's ability to judge time durations accurately declines with advancing age. Wearden (2005), for instance, demonstrated that older people tend to experience time periods to be of shorter duration (time to pass faster) than do youngsters and middle-aged people. In another meta-analysis, Block et al. (2000) demonstrated the presence of gender differences in the accurateness of time duration judgments with females judging durations less precisely compared to males. Another characteristic is task involvement, that is, whether or not respondents are familiar with and/or involved in the choice task. lyengar and Lepper (2000), for 
instance, argued that people who are not familiar or have not wellidentified prior preferences with choice subjects will experience more difficulties when looking for options that match their own preferences. One possible intuitive reasoning is that this may possibly negatively impact the accuracy of the people's perceptions of time durations. ${ }^{3}$ To control for these possible influences and to limit the possibility for omitted variable bias in the estimations, the present study accounts for gender and age in the MLM estimations. ${ }^{4}$

\section{3 | CHOICE TASKS}

The procedure to collect the data is in many respects similar to the one used in several previous studies. The choice tasks were conducted in computer laboratories at the university with first-year students of the undergraduate study program in Business Administration at the University of Leuven (Belgium). The sessions were organized during official teaching hours (i.e., $2 \mathrm{hr}$ was scheduled in the curriculum). The students were told that participation implied that one would have a chance of winning a cinema ticket (value of €9) in a draw. Upon arrival at the computer laboratory, participants were informed about the guidelines of the choice tasks. The actual objective of the study was not communicated to the participants. Participants were told only that the purpose of the choice tasks was to study the choice-making behavior of people. Regarding the setting and instructions of the choice tasks, participants were provided with some explanatory information on a computer screen before starting the choice tasks. For instance, participants were informed that they were about to be asked to participate in a series of choice tasks and that the objective of the choice tasks was to rank the options in the choice set according to their preference of choice, that is, the most preferred alternative first, the second most preferred option second, and so on. Participants were also explicitly told that the ordering of the choice options in the choice matrix was arbitrary. Each participant was asked to complete the choice tasks on an individual basis. Two supervisors were present to see to it that no interaction was possible between the participants. The software used to perform the choice tasks automatically registered the time spent by participants on performing the choice tasks. For each choice set, participants were asked to estimate the amount of time spent in the choice process.

A series of choice tasks that involved five different choice stimuli were presented to the participants: booking a holiday hotel, booking a restaurant, selecting a partner for a work project, buying a smartphone, and choosing a job. For each choice task, a hypothetical choice context was designed. For instance, for the choice task that

${ }^{3}$ Another possible reasoning (as correctly noted by an anonymous referee) is that people who are more involved with choice tasks are more likely to adopt a maximizing choice-making strategy compared to their counterparts, on average.

${ }^{4}$ Another personal characteristic that has been found to be related to the accuracy of the people's perceptions of time durations is education background or level (Coelho et al., 2004). Given that the present study used data that were solely obtained from students in the same education program and, hence, with largely similar education background or level, the MLM analysis did not control for this factor. However, in case of a sample with participants of mixed education backgrounds, it would be important to control for education background or level in the regressions. involved ranking holiday hotels, participants were asked to book a holiday hotel via the website of a popular tour operator. The participants were told that the choice matrix displayed descriptions of the hotel amenities and room features as collected from this website. The choice tasks differed in that either the number of choice options in the choice set was altered (with the number of options in the choice set varying from 5 to 7 to 10 choice options), the amount of choice information as measured by the number of attributes for which product information is available being different (the number of attributes ranging from 5 to 7 to 10 attributes), or a combination of both. As mentioned previously, the choice for the five particular choice stimuli (holiday hotel, smartphone, restaurant, working partner, and job) was driven by the aim of selecting choice tasks that (also in real-life situations) require the average individual to spend considerable cognitive effort, deliberation, and time (contrary to day-to-day decisions that are done in some cases almost instantaneously or at least without spending several minutes deciding on what product to buy). The idea behind choosing choice tasks that involved respectively 5,7 , and 10 choice options and 5, 7, and 10 attributes with choice information was to design choice tasks that vary from a low difficulty level to medium difficulty level to high difficulty level. Note that a similar number of choice options and attributes were also used by some previous studies (DeShazo \& Fermo, 2002; lyengar \& Kamenica, 2010; Snibbe $\&$ Markus, 2005). ${ }^{5,6}$

In the design of the choice tasks, the idea was to use real data wherever possible. ${ }^{7}$ As such, the choice options, the set of attributes, and attribute information were obtained through an analysis of commercial sources and free elicitation. For the attributes for which no real values were available, realistic values were defined. For the selection of the choice options and the attributes for the choice task about smartphones, a website with detailed reviews of smartphones was consulted. $^{8}$ The job descriptions were collected from a job vacancy website. The data on restaurants were obtained from a website with reviews for restaurants in Belgium. For the attributes used in the task on partner choice for a work project, a combination of data collected from curriculum vitae and hypothetical data was used. In all choice

${ }^{5}$ DeShazo and Fermo (2002) employed four to nine choice options and two to nine attributes in their analysis of choice tasks. lyengar and Kamenica (2010) used three options (limited condition) and 11 options (extensive condition). Snibbe and Markus (2005) used 10 choice options in a marketing research study. Note, however, that there are also other studies that used larger numbers (e.g., lyengar \& Lepper, 2000).

${ }^{6} \mathrm{An}$ anonymous referee pointed out that that one could equally argue in favor of distinguishing only between low difficulty level and high difficulty level instead of low difficulty level versus medium difficulty level versus high difficulty level. An alternative would be to distinguish between choice tasks of low difficulty level versus medium difficulty level versus high difficulty level, however using a better spread between the number of choice attributes and/or alternatives (e.g., 3 alternatives vs. 8 alternatives vs. 13 alternatives).

${ }^{7}$ Note that the aim to design choice tasks as much as possible in correspondence with real choice tasks and capture some variety in the number of choice features and choice alternatives (without ending up with too much choice tasks for the respondents to solve) somewhat reduced consistency across the choice tasks in terms of the number of choice attributes and the number of choice options.

${ }^{8}$ See Appendix A for an example of a choice task that was used in the present study (choice tasks for the smartphone with five choice options and seven attributes with choice information). 
TABLE 1 Features of the choice tasks

\begin{tabular}{lllll} 
Choice task & Choice subject & Number of attributes & Number of choice options & Attributes $\times$ Choice Options \\
\hline 1 & Holiday hotel & 5 & 5 & 25 \\
2 & Holiday hotel & 5 & 10 & 50 \\
\hline 3 & Restaurant & 5 & 7 & 35 \\
\hline 4 & Smartphone & 7 & 5 & 35 \\
\hline 5 & Partner & 5 & 5 & 5 \\
\hline 6 & Partner & 10 & 5 & 50 \\
\hline 7 & Job & 7 & 7 & 49 \\
\hline 8 & Job & 10 & 10 & 100 \\
\hline
\end{tabular}

tasks, choice options were presented without pictures and/or (brand) names to avoid that choices would be driven by visual aspects and/ or the brand image (e.g., a too strong familiarity with one particular brand could result in the choice maker using a highly selective choice-making process, impacting the duration judgment). Qualitative data retrieved from real data sources were, if needed, transformed into ratings on a 10-point rating scale. Note that all attributes were formulated such that higher attribute values are desirable. The features for the choice tasks are summarized in Table 1.

The survey also included some questions to collect some personal information of the respondents (age, gender, and task involvement). Several items were included to gauge the respondents' familiarity and involvement with the choice tasks. For instance, for the choice task about choosing a smartphone, respondents were asked whether or not they have a smartphone. In the choice tasks about selecting a restaurant, the participants were asked to indicate how frequently they visit a restaurant on a monthly basis.

\section{I MAXIMIZING TENDENCY SCALE}

During the session with choice tasks, students were also asked to complete the Maximizing Tendency Scale (MTS) of Diab, Gillespie, and Highhouse (2008). This is only one of the measurement scales that have been developed in the maximization literature to measure people's tendency to maximize in choice and decision making. In fact, building on Herbert Simon's $(1955,1956)$ work, Barry Schwartz et al. (2002) were the first to further elaborate on the maximizing and satisficing orientations in choice and decision making. This resulted in, among other things, the development of the Maximizing Scale (MS). Other scales that have been developed include the short form of the MS of Nenkov et al. (2008), the MTS of Diab et al., the Maximization Inventory Scale of Turner, Rim, Betz, and Nygren (2012), the seven-item MTS of Dalal et al. (2015), and the Decision Making Tendency Inventory of Misuraca, Faraci, Gangemi, Carmeci, and Miceli (2015; for an overview of the measurement scales, see Cheek \& Schwartz, 2016). Within the maximization literature, there is a debate regarding the proper measurement of the maximizing tendency (Cheek \& Schwartz, 2016). One of the main reasons for the lack of consensus is that several conceptualizations and measures of maximization have emerged in the literature. Nevertheless, in spite of this lacking consensus, several studies (e.g., Nenkov et al., 2008) have demonstrated that the MTS of Diab et al. is conceptually and psychometrically more valid than the (more commonly used) MS of Schwartz et al. In the present analysis, the MTS was employed in the measurement of the participants' maximizing tendency. ${ }^{9}$ This MTS measurement scale comprises nine items with Likert scales to measure people's tendency to maximize in choice and decision making, with higher ratings indicating a higher maximizing tendency (for the exact items and the psychometric properties of the MTS measurement scale, see the original paper). ${ }^{10}$ In particular, by summing the ratings on the nine comprising items, the authors obtained a summative MTS score is (range of the MTS scores: 7-63). Whereas most previous studies (Dar-Nimrod et al., 2009; Misuraca \& Teuscher, 2013; Schwartz et al., 2002) modeled the tendency to maximize solely as a discrete variable in the analysis (typically by performing a median split based on the summative maximization score), the present study uses both continuous and discrete versions of the maximizing tendency. As a continuous measure of the participants' tendency to maximize, the summative MTS score is used. As a discrete measure of maximizing tendency, the 75 th and 25 th percentile value split and the 90th and 10th percentile value split are performed. The idea is that this enables distinguishing between people who have a rather strong habit of maximizing and people who are much less prone to maximizing (75th and 25the percentile split) and between people who have a very strong tendency to maximize and people who almost do not have this tendency (90th and 10th percentile split) in choice making.

\section{5 | DURATION JUDGMENT RATIO}

Most studies use duration judgment ratios to measure the discrepancy between the estimated and actual time duration (e.g., Block et al., 2010; Block, Zakay, \& Hancock, 1999). In the context of people performing choice tasks, such a ratio would be computed as the ratio of actual versus estimated choice time (Misuraca \& Teuscher, 2013). In the interpretation of such ratio values, values greater than 1 are considered to indicate an overestimation of the actual choice time. The opposite interpretation holds for ratio values lower than 1: an

\footnotetext{
${ }^{9}$ As some kind of (simple) robustness test, MLMs were performed with the MS of Schwartz et al. (2002) and the Maximization Inventory scale of Turner et al. (2012). The MLM analysis generated similar results.

${ }^{10}$ All nine items of the MTS were measured using 7-point Likert scales ranging from 1 (completely disagree) to 7 (completely agree).
} 
underestimation of choice time by the choice maker. Ratio values near to or equal to 1 imply that choice makers nearly perfectly judged choice time.

In the present analysis, due to the specificities of the MLM approach, the use of a normalized version of the duration judgment ratios is more practical. More precisely, as the purpose of the study is to analyze the accuracy of peoples' duration judgments of choice time, the accuracy measure should enable distinguishing between accurate and inaccurate duration judgments of choice time. The use of the traditional duration judgment ratio in an MLM does not enable such a distinction in a straightforward manner as both values lower than 1 and higher than 1 indicate inaccurate duration judgments of choice time. To deal with this issue, duration judgment ratios are normalized. Set estimated choice time equal to $t_{\text {est }}$ and actual choice tome equal to $t_{\text {act }}$; then

$$
\begin{aligned}
& \text { if } t_{\mathrm{est}}>t_{\mathrm{act}} \rightarrow \text { normalized duration ratio }=t_{\mathrm{act}} / t_{\mathrm{est}} \\
& \text { if } t_{\mathrm{est}}=t_{\mathrm{act}} \rightarrow \text { normalized duration ratio }=1 ; \\
& \text { if } t_{\mathrm{est}}<t_{\mathrm{act}} \rightarrow \text { normalized duration ratio }=t_{\mathrm{est}} / t_{\mathrm{act}} .
\end{aligned}
$$

The normalized version of the duration judgment ratio can take values between 0 and 1 . It holds that values equal to 1 indicate a perfectly accurate duration judgment of choice time. Values lower than 1 indicate that the respondent perceived the time spent in the choice task inaccurately. The lower the normalized duration ratio, the more inaccurate the duration judgment. In the empirical analysis below, the normalized duration ratio is the dependent variable. As noted, whereas the use of this normalized duration judgment ratio is more practical in the MLM setting, the drawback is a loss of information as it is no longer possible to determine whether inaccurate duration judgments are caused by an underestimation or overestimation of choice time.

\section{6 | DATA}

The sample set includes choice data of 309 students. The number of respondents is roughly equally divided between males and females (57.6\% vs. $43.4 \%$ ). The mean age of the participants was 21.28 years ( $S D=0.30$ years). On the basis of the answers collected on the questionnaire items measuring choice task involvement, an involvement dummy was constructed (dummy value equal to 1 indicating that respondents were generally involved with the five choice tasks in the present study). The involvement data show that between $80 \%$ and $90 \%$ of the respondents are familiar with the choice tasks (exact percentages varying across the choice tasks).

As to the tendency of the participants to maximize in choice making, the descriptive statistics of the MTS scores show that the participants vary widely in their tendency to strive for the best outcome in choice making. More precisely, the descriptive statistics (MMTS score $=41.1, S D=7.91, M d n=41)$ of the summative MTS scores suggest distribution curves that are more or less bell shaped. The high maximum and 90the percentile MTS values of respectively 63 and 51 indicate the presence of some participants who are rather compulsive maximizers and the low minimum, and 10th percentile MTS values of respectively 17 and 31 suggest the presence of some participants who almost feel no (or at least much less) need to maximize in choice making. The middle group with summative MTS scores between the 25 th percentile $($ MTS value $=36$ ) and 75 th percentile $($ MTS value $=47$ ) represent the large group of participants who are positioned between these two extremes, that is, adopting a more mixed-choice orientation.

The descriptive statistics for the time spent in the choice task and the normalized duration judgment ratios are shown in Table 2. The descriptive statistics for the time spent in the choice task (time in seconds) show two interesting preliminary findings. First, for most of the choice subjects, it holds that average choice time increases as the choice task becomes more complex. Second, the high values for the standard deviations and the large differences between the minimum and maximum values show for each choice task strong differences in choice time between respondents. The average normalized duration judgment ratios indicate that on average estimated choice time deviates approximately $30 \%$ of actual choice time across the choice tasks. This suggests that the average participant is rather inaccurate in the perception of choice time. As shown by the large standard deviation values and the quartile values, the accuracy of the choice time estimates varies substantially among respondents and across choice tasks. Moreover, the small minimum values denote that some participants performed very poorly in estimating the time spent on choosing. On the other hand, the maximum values suggest that some participants succeeded in judging choice time almost perfectly. However, in general, the descriptive statistics for the normalized duration judgment ratios show that people are not able to discern the precise choice time duration. This suggests that people are prone to underinvesting or overinvesting time in choice making.

\section{7 | RESULTS}

The MLM technique enables accounting for the repeated measure structure in the data with the same sample of participants completing multiple choice tasks (estimations were performed by the software package MLwiN Version 2.25). Due to this nested structure, part of the variation in the normalized duration judgment ratio is being situated at the level of the participants and part at the level of the choice tasks. The MLM analysis proceeds in a stepwise manner in which choice task features and respondent characteristics are consecutively introduced into the model. The results are listed in Table 3.

As a baseline model, the null model is computed to obtain an estimate of the basic partitioning of the variation in the normalized duration judgment ratios among the levels of the participants and the choice tasks. The results are listed in the first column of Table 3. The coefficient of .689 indicates that the average respondent misjudged choice time across choice tasks with approximately $30 \%$. As to the basic partitioning of the variance, results suggest that roughly a quarter of the variance in the normalized duration judgment ratios $(26.8 \%$ of the variance) is situated at the level of the participant and approximately three quarters are situated at the level of the choice tasks (73.2\% of the variance). Both variance components are statistically significant at the .001 level. These results suggest that choice task 
TABLE 2 Descriptive statistics of the time spent in the choice experiments and duration judgments of choice time after normalization

\begin{tabular}{|c|c|c|c|c|c|c|c|}
\hline Choice task & Average & SD & Minimum & 25th percentile & Median & 75th percentile & Maximum \\
\hline \multicolumn{8}{|c|}{ Time spent in the choice experiments } \\
\hline 1 & 98.08 & 40.07 & 20.62 & 71.82 & 91.89 & 117.08 & 291.32 \\
\hline 3 & 130.03 & 73.32 & 14.44 & 93.38 & 116.96 & 155.37 & 946.36 \\
\hline 4 & 71.14 & 34.89 & 12.11 & 49.27 & 65.38 & 85.03 & 378.95 \\
\hline 7 & 112.31 & 73.05 & 13.38 & 75.89 & 97.42 & 128.06 & 726.20 \\
\hline 8 & 273.39 & 180.09 & 10.47 & 172.15 & 242.25 & 334.31 & 2016.12 \\
\hline \multicolumn{8}{|c|}{ Duration judgments of choice time after normalization } \\
\hline 1 & 0.682 & 0.208 & 0.080 & 0.534 & 0.700 & 0.849 & 0.999 \\
\hline 6 & 0.654 & 0.210 & 0.142 & 0.498 & 0.660 & 0.804 & 0.998 \\
\hline 7 & 0.655 & 0.203 & 0.146 & 0.505 & 0.679 & 0.823 & 0.992 \\
\hline 8 & 0.765 & 0.169 & 0.070 & 0.579 & 0.718 & 0.869 & 1.000 \\
\hline
\end{tabular}

Note. Time spent in the choice experiments is in seconds; normalized duration judgment ratios are dimensionless.

features and participant characteristics play a significant role in explaining the variance in the accuracy of the participants' judgments of choice time. ${ }^{11}$

In the ensuing models (Models 1 to 3 ), choice task features and participant characteristics are gradually introduced in the MLM analysis under the assumption of coefficients being fixed, that is, fixed effects. As choice task features, the following variables are included in the model: the number of options in the choice set (baseline category: five choice options), the number of attributes on which the choice options are described (baseline category: five attributes), and the product of the number of attributes and the number of choice options (Attributes $\times$ Options). Four personality features of the participants are included: age, gender, a measure of task involvement, and a measure of the participant's tendency to maximize in choice and decision making. Whereas gender, age, and the measure of task involvement are introduced as control variables to limit the possibility for omitted variable bias in the MLM estimations, the measure of the participants' tendency to maximize is the personality feature of interest given the focus of the present research paper.

Model 1 includes the summative MTS score as derived from the MTS of Diab et al. (2008) as a continuous measure of the participants' affinity with maximizing in choice and decision making. In Models 2 and 3 , discrete versions of the MTS score are used to distinguish between people with different tendencies to maximize in choice and decision making. Model 2 distinguishes between people who have a habit of maximizing and people who are much less prone to maximizing (75th and 25the percentile split) relative to the people with a more

\footnotetext{
${ }^{11}$ As requirements for the MLM approach, the basic assumptions of linearity and normality were tested using the residuals of the baseline model. An inspection of the normal scope and catch-all plots of the residuals revealed that both assumptions generally hold quite well (for more, see, among others, Hox, 2010).
}

mixed strategy in choice and decision making (baseline category: 25th percentile < MTS score < 75th percentile). Model 3 looks at how people who feel a very strong need to maximize (MTS score $>90$ th percentile) and people who almost do not experience such a need (MTS score < 10th percentile) perform in judging choice time accurately relative to people with a less outspoken, more mixed orientation (baseline category: 10th percentile < MTS score < 90th percentile).

The results of Models 1-3 show some interesting findings. First, the negative coefficient estimates (statistically significant at $p<.001$ ) for the variables measuring the number of attributes with product information, the number of options in the choice set, and the combination of both (the interaction term Attributes $\times$ Options) show that as more choice options and/or more choice information are available, people do worse in judging choice time correctly. Note that the negative relationship is stronger as more choice options are included in the choice set and more choice information is available. This result corresponds to the finding of Fasolo et al. (2009) that the number of choice options and the amount of choice information available affect the accurateness of people's perception of choice time. This negative coefficient estimate seems also in line with the stipulations of the attentional resource theory (e.g., Kahneman, 1973) that as choice tasks involve more choice options and/or more choice information, it is necessary for the choice maker to allocate more cognitive and attentional effort to the actual screening of the choice information and selecting a final choice. As a consequence, less cognitive resources are available for keeping track of the actual time spent in choice making. Note that this pattern of more choice freedom and more choice information trading off with less accurate perceptions of the choice time is observed for participants irrespective of their tendency to strive for the best outcome in choice making. More indirectly, the finding of a statistically significant negative relation confirms what is referred to in the academic literature as the 
TABLE 3 Multilevel modeling analyses of the (normalized) duration judgments of choice time

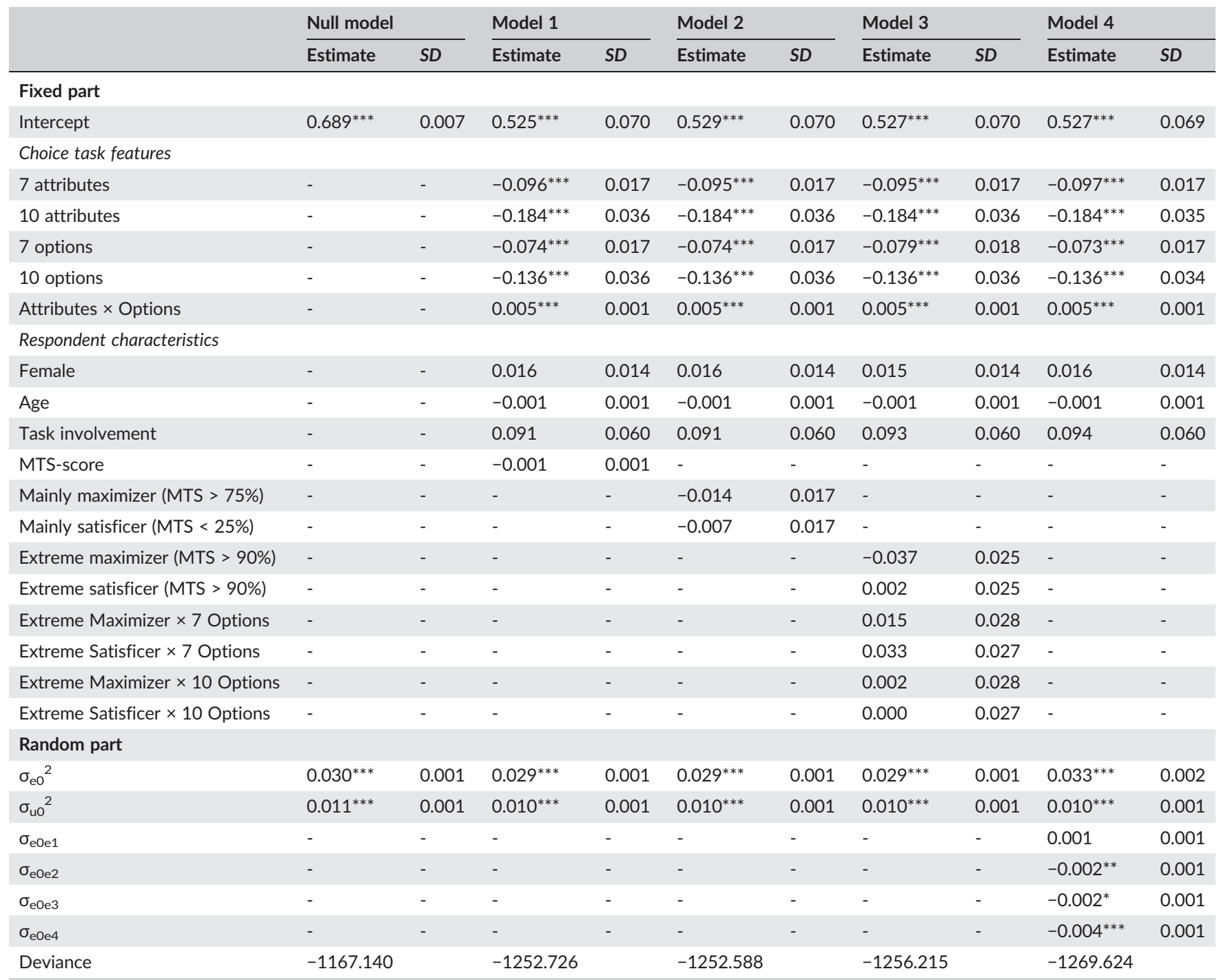

Note 1. $\sigma_{\mathrm{e} 0}{ }^{2}=$ within-participant, between choice task variance in the (normalized) duration judgments of choice time; $\sigma_{\mathrm{u} 0}{ }^{2}=$ between-participant variance in the (normalized) duration judgments of choice time; $\sigma_{\mathrm{e} 0 \mathrm{e} 1}$ and $\sigma_{\mathrm{e} 0 \mathrm{e} 2}=$ covariance terms between the variables measuring the number of attributes with choice information and the (normalized) duration judgments of choice time (seven attributes and 10 attributes, respectively, vs. the baseline category of five attributes); $\sigma_{\mathrm{eOe} 3}$ and $\sigma_{\mathrm{eOe} 4}$, covariance terms between the variables measuring the number of options in the choice set and the (normalized) duration judgments of choice time (seven options and 10 options, respectively, vs. the baseline category of five options).

Note $2:^{* * *},{ }^{* *}$ and ${ }^{*}$ denote, respectively, significance at $1 \%, 5 \%$ and $10 \%$ level.

the dashes indicates cells that should be blank.

"paradox of choice" or "excessive choice effect" (Schwartz, 2004), that is, more choice freedom and choice information hampering good choice making. As to the control variables measuring gender and age, the model estimates show no significant difference between males and females and between younger and older participants in judging choice time accurately. Both findings contrast with the findings of previous papers (e.g., Block et al., 2000; Wearden, 2005). ${ }^{12}$

\footnotetext{
${ }^{12}$ Possible explanations for the finding of insignificant relationship for gender and age are that the present analysis controls for several choice task features as well as for people's tendency to maximize (typically not done in previous studies focusing on gender and age in the judgment on time durations). Another possible explanation is that only undergraduate students participated in the present study. This makes it so that only a limited age range is covered. A similar study involving participants from all age groups could very well yield different results. It is up to future research to examine this relationship further.
}

As to the task involvement of the respondents, the MLM results suggest a positive yet statistically insignificant relation to the accuracy in judging time spent in the choice task accurately. Whereas the positive association is consistent with the intuition that people who are familiar with choice tasks experience less difficulties when looking for options that match their own preferences, thereby making it easier to keep track of time spent in performing the choice task, the results of the present study cannot confirm the statistical significance of this association.

The coefficient estimates for the maximizing tendency of the participants in Models 1-3 consistently show that the participants' tendency to maximize in choice making does not relate statistically significantly to the accurateness of people's duration judgments of choice time (e.g., $p$ value of .334 for the continuous MTS score). Overall, these results disconfirm the, which states that people with a (very) 
outspoken tendency to maximize are less accurate in judging choice time after controlling for several relevant features of the choice task and the participant. As a possible explanation for this finding of a statistically nonsignificant relationship, there is an argument put forward in the literature on constructive decision- and choice-making processes (e.g., Bettman et al., 1998; Biehal \& Chakravarti, 1986) that a large majority of the people do not have a dominant choice strategy (such as maximizing or satisficing). Instead, this literature argues that people use multiple choice-making strategies (e.g., elimination-byaspects choice strategy, confirming dimension choice strategy, and lexicographic choice strategy), with the choice for pursuing a particular choice-making strategy being highly contingent on the specific choice task and context. However, whereas there are quite some qualitative studies that provided support for the postulations of this theory on constructive decision processes (Bettman et al., 1998; Biehal \& Chakravarti, 1986), only limited quantitative empirical evidence has been accumulated. Nevertheless, in the present study, this reasoning could possibly explain for why participants with (very) high, moderate, and (very) low summative MTS scores for maximizing tendency are more or less equally inaccurate in judging choice time. In particular the ideas of constructive choice-making processes could imply that participants with high MTS scores adopted a maximizing approach for solving some of the choice tasks (e.g., choice tasks involving certain choice stimuli) yet other choice-making approaches for performing the other choice tasks. In the same vein, participants with low MTS scores may have applied a satisficing approach in some choice tasks but other choice-making strategies in the other choice tasks. At least further quantitative research is needed to confirm this reasoning.

To test, which states that the presence of less (more) choice options and/or less (more) choice relevant information will attenuate (appreciate) the difference in the accuracy of the duration judgments of choice time between maximizers and satisficers, interaction terms were added to the MLM (Model 3 in Table 3 ). It concerns interaction terms between the discrete version of the MTS score identifying participants with a strongly outspoken maximizing tendency and participants with (nearly) no such tendency (participants with MS score > 90th percentile value and participants with MTS score $<10$ th percentile value, respectively) and the number of choice options offered (Extreme Maximizer $\times 7$ Options, Extreme Satisficer $\times 7$ Options, Extreme Maximizer $\times 10$ Options, and Extreme Satisficer $\times 10$ Options). All interaction terms turned out to be statistically insignificant. Though not reported in Table 3, statistically insignificant coefficient estimates were also found for the interaction terms between the discrete version of the MTS score identifying the same group of participants and the number of attributes with choice information. At first sight, these findings disconfirm . As noted above, as a possible intuitive explanation, there is the idea discussed in the theory of constructive decision and choice making (Bettman et al., 1998; Biehal \& Chakravarti, 1986) that a large majority of the people do not have a dominant choice-making strategy.

In the last step of the analysis, the MLM was modified such as to allow heterogeneity in the study of the associations between the choice task features and the accurateness of participants' duration judgments of choice time. In particular, the assumption of coefficients being fixed was relaxed. This enables estimation of the variance and covariance terms for the variables measuring the choice task features. The results of this analysis are listed in Model 4 in Table 3. The estimations denote some interesting insights. First, the statistically significant variance coefficient $\sigma_{\mathrm{e} 0}{ }^{2}$ indicates that, in general, the preciseness with which participants judge the duration of the choice time varies considerably across the choice tasks. A second interesting observation is that the between-participant variance $\sigma_{\mathrm{uo}}{ }^{2}$ is also statistically significant. This suggests that there is a lot of variation between participants in terms of how accurately they perceive choice time. Third, the covariance terms for the variables measuring the number of attributes with available product information $\left(\sigma_{\mathrm{e} 0 \mathrm{e} 1}\right.$ and $\left.\sigma_{\mathrm{eOe} 2}\right)$ are both statistically insignificant. Fourth, and different to the third finding, covariance terms for the variables measuring the number of options in the choice sets $\left(\sigma_{\mathrm{eOe} 3}\right.$ and $\left.\sigma_{\mathrm{eOe} 4}\right)$ are both statistically significant and negative. Explaining the latter two results is not straightforward. One possible explanation could be that as the number of choice options increases, participants become increasingly similar in terms of judging choice time (in)accurately. The diminishing differences in the duration judgment ratios could very well be explained by the ideas of the aforementioned theory of constructive decision and choice making. More precisely, it could be that as the choice task involves a higher number of choice options, participants, irrespective of their tendency to maximize, switch to other choicemaking strategies (e.g., elimination-by-aspects choice strategy). Further research could help to unravel what exactly explains these findings.

\section{I CONCLUSIONS AND POLICY IMPLICATIONS}

The main objective of this study was to learn more about how the accuracy of people's perception of choice time varies with different choice task features and personal characteristics of the choice maker. A special focus was on two key research questions. A first research question was whether the precision of people's duration judgments of choice time relates to the number of choice options and/or the amount of choice information. A second key research question was whether maximizers and satisficers differ in terms of the accuracy of their duration judgments of time spent in the choice task. On the basis of a review of previous psychological and maximization literature, hypotheses were formulated. To test these hypotheses, a series of choice tasks that (a) included either a relatively low, moderate, and high number of options in the choice set; (b) provided the choice maker with a relatively low, moderate, or large amount of choice information; and (3) involved different types of choice stimuli (booking a holiday hotel, booking a restaurant, selecting a partner for a work project, buying a smartphone, and choosing a job) were designed. A sample of 309 undergraduate students was asked to complete the choice tasks. An MLM analysis approach was used to account for the repeated-measure structure, with respondents completing multiple choice tasks.

Regarding the choice task features, the MLM results demonstrated that people are less accurate in estimating choice time as 
choice tasks offer more choice freedom (a larger number of choice options) and/or more choice information. This suggests that increasing the amount of choice information or expanding the number of choice options beyond a certain threshold generates considerable cognitive burden to the choice maker, which results in the choice maker having less accurate perceptions of the time spent in choice making. At first sight, this finding seems consistent with the theoretical models as discussed in the psychology research literature (e.g., the attentional resource theory, see Block \& Zakay, 2008; Kahneman, 1973), which, broadly speaking, postulates that more complex choice and decision problems make it more difficult for people to keep track of time as fewer attentional resources are available for temporal information processing. So, even though providing people with more complete descriptions of the available choice options is what conventional wisdom advocates, it is not clear-cut as to whether the net effect of more detailed presentations of choice options is actually positive to the average individual.

As to the role of people's tendency to maximize in choice and decision making, it was hypothesized that people with a higher tendency to maximize in choice making will judge choice time more inaccurately as compared to people who experience a lower need to look for the best choice option. The reasoning for this hypothesis was that maximizers, due to their tendency to look for the best choice option, adopt more exhaustive search and choice-making procedures and as such are more prone to have less attentional resources at their disposal for keeping track of choice time. MLM results demonstrated, however, contrary to what was hypothesized, that participants with a (strong to very strong) tendency to maximize in choice making do not differ significantly in estimating choice time accurately as compared to the participants with a less outspoken tendency to maximize. In fact, results indicated that people with a high tendency to maximize in choice making are more or less equally inaccurate in perceiving choice time as compared to people who experience a low to very low motivation to look for the best option in choice and decision making. Although the paper did not provide a concise explanation for this finding, it was argued that a possible explanation could be found in the literature on constructive decision- and choice-making processes (e.g., Bettman et al., 1998; Biehal \& Chakravarti, 1986). This literature argues that a large majority of the people do not have a dominant choice strategy (such as maximizing or satisficing). Instead, people employ multiple choice-making strategies (e.g., eliminationby-aspects choice strategy and lexicographic choice strategy), the choice for pursuing a particular choice-making strategy being highly contingent on the nature and the specificities of the choice task and context.

Although one should be cautious in generalizing the findings of the present study because the student population might not be representative for the general public, the results do provide preliminary evidence that increasing the number of choice options, the amount of choice information, or the combination of both relates negatively to how accurately people judge choice time, irrespective of their tendency to maximize in choice making. Although this result is surely interesting from a research point of view, one important question is whether it may be a source of concern in practice. The answer to this question is clearly that it is. Even more so if future studies would replicate the present findings with the general public. More precisely, whereas there are quite some studies in the behavioral and experimental economics literature that say that the provision of more information improves choice accuracy and boost the uptake of some policies (Allcott, 2011; Allcott \& Rogers, 2014), the findings of the present study suggest that the impact of providing people with a lot of choice options and choice information may be more complex than accounted for in these analyses. The present results suggest, for instance, that choice architects should bear in mind that when people are overloaded with choice options and choice information (increasing choice complexity), they are prone to lose track of choice time and misallocate time to choice processes. The result can be that they make suboptimal choice decisions (hence causing a welfare loss) by overinvesting or underinvesting time in choice and decision making. Therefore, when designing choice sets, choice architects should aim for a choice task design that presents people with sufficient choice freedom and choice information (to make people better informed to choice making) yet without providing them with information that is overly complex and too cognitively demanding. Note that finding such a balance is particularly relevant given the findings of previous studies that people are sensitive to time costs in choice making, people's shopping behavior is strongly influenced by perceived time spent shopping (e.g., Ackerman \& Gross, 2003; Mantel \& Kellaris, 2003), and people exit the choice process when they feel they have reached their personally and implicitly determined time limit for making choices. Nevertheless, further research is needed to obtain more accurate and reliable insights as to what is a reasonable number of options and amount of information.

It is important to note that the present study suffers from some limitations. Perhaps the most important limitation is that the data on the choice tasks were solely obtained from students in the same education program, and hence, with largely similar age and education background. As mentioned above, most likely, this sample of students is not representative of the general public, which makes it so that one should be very cautious in generalizing the results. In order to obtain more general results, it would be interesting to replicate the present study with the general public. A second limitation is that not all potentially influential choice task features (e.g., time pressure and task familiarity) and personal characteristics (e.g., emotional involvement, memory capacity, and cultural background) were accounted for in the analyses. Therefore, an interesting topic for further research would be to expand the present study to other choice contexts and choice stimuli, thereby accounting for a broader set of task features and personal characteristics. A third shortcoming is that choice tasks studied in the present paper are not completely analogous to real-world choice-making settings. For instance, real-world choice tasks do not require (at least not explicitly) people to rank the available choice options before making the final choice. Also, whereas the present choice tasks provide all choice information in overview tables, in the real world, more often than not the choicemaking context is more complex and disorganized, with information on some choice options not or only partially being available, choice information not always being objective, and so on. This makes it so that it is typically more cognitively demanding for people to collect and process the choice information on the different choice options. 
This disorganization of choice information may impact the accurateness of the perceptions of choice time. A fourth potential issue is that the present study includes only a measure of the respondents' general tendency to search for the absolute best in choice making As such, the present study can only look at how the respondents' general maximizing tendency relates to the accuracy in judging time spent in choice making accurately across the different choice tasks. There is, however, empirical evidence suggesting that people's tendency to maximize (or satisfice) relates to the choice task and context (e.g., Schwartz, 2004). Therefore, it would be interesting to explore how the respondents' accuracy in judging the choice time accurately relates to their tendency to maximize in the particular choice task. Note that this requires a measure of maximizing tendency that is specific for each choice task and, hence, probably a more qualitative analysis approach. Another topic that warrants further investigation is a comparison of the exact choice-making mechanisms used by people with different tendencies to maximize. For instance, with the ideas of the constructive decision and choice-making theory (e.g., Bettman et al., 1998; Biehal \& Chakravarti, 1986) in mind, it would be interesting to explore whether or not, and if so, how and when, people with a high tendency to maximize consider switching to other choice-making strategies as the cognitive load of the choice task increases.

\section{ACKNOWLEDGEMENTS}

I thank the editor of the journal and the anonymous referees for very helpful comments and suggestions to this or an earlier version of the paper.

\section{ORCID}

Nicky Rogge $\bigcirc$ http://orcid.org/0000-0001-7755-0471

\section{REFERENCES}

Ackerman, D. S., \& Gross, B. L. (2003). So many choices, so little time: Measuring the effects of free choice and enjoyment on perception of free time, time pressure and time deprivation. Advances in Consumer Research, 30, 290-294.

Allcott, H. (2011). Social norms and energy conservation. Journal of Public Economics, 95(9), 1082-1095.

Allcott, H., \& Rogers, T. (2014). The short-run and long-run effects of behavioral interventions: Experimental evidence from energy conservation. The American Economic Review, 104(10), 3003-3037.

Arunachalam, B., Henneberry, S. R., Lusk, J. L., \& Norwood, F. B. (2009). An empirical investigation into the excessive-choice effect. American Journal of Agricultural Economics, 91(3), 810-825.

Besharat, A., Ladik, D. M., \& Carrillat, F. A. (2014). Are maximizers blind to the future? When today's best does not make for a better tomorrow. Marketing Letters, 25, 77-91.

Bettman, J. R., Luce, M. F., \& Payne, J. W. (1998). Constructive consumer choice processes. Journal of Consumer Research, 25(3), 187-217.

Biehal, G., \& Chakravarti, D. (1986). Consumers' use of memory and external information in choice: Macro and micro perspectives. Journal of Consumer Research, 382-405.

Block, R. A. (2003). Psychological timing without a timer: The roles of attention and memory. In H. Helfrich (Ed.), Time and mind II: Informationprocessing perspectives (pp. 41-59). Seattle, WA: Hogrefe \& Huber.
Block, R. A., Hancock, P. A., \& Zakay, D. (2000). Sex differences in duration judgments: A meta-analytic review. Memory \& Cognition, 28 (8), 1333-1346.

Block, R. A., Hancock, P. A., \& Zakay, D. (2010). How cognitive load affects duration judgments: A meta-analytic review. Acta Psychologica, 134(3), $330-343$

Block, R. A., \& Reed, M. A. (1978). Remembered duration: Evidence for a contextual-change hypothesis. Journal of Experimental Psychology: Human Learning and Memory, 4, 656-665.

Block, R. A., \& Zakay, D. (1997). Prospective and retrospective duration judgments: A meta-analytic review. Psychonomic Bulletin \& Review, 4 (2), 184-197.

Block, R. A., \& Zakay, D. (2008). Timing and remembering the past, the present, and the future. In S. Grondin (Ed.), Psychology of time (pp. 367-394). Bingley, England: Emerald.

Block, R. A., Zakay, D., \& Hancock, P. A. (1999). Developmental changes in human duration judgments: A meta-analytic review. Developmental Review, 19(1), 183-211.

Boatwright, P., \& Nunes, J. C. (2001). Reducing assortment: An attributebased approach. Journal of Marketing, 65(3), 50-63.

Botti, S., \& lyengar, S. S. (2006). The dark side of choice: When choice impairs social welfare. Journal of Public Policy \& Marketing, 25(1), 24-38.

Botti, S., \& McGill, A. L. (2006). When choosing is not deciding: The effect of perceived responsibility on satisfaction. Journal of Consumer Research, 33(2), 211-219.

Brown, S. W. (1997). Attentional resources in timing: Interference effects in concurrent temporal and nontemporal working memory tasks. Perception \& Psychophysics, 59(7), 1118-1140.

Brown, S. W., \& Boltz, M. G. (2002). Attentional processes in time perception: Effects of mental workload and event structure. Journa of Experimental Psychology: Human Perception and Performance, 28(3), 600.

Brown, S. W., \& West, A. N. (1990). Multiple timing and the allocation of attention. Acta Psychologica, 75(2), 103-121.

Bruine de Bruin, W., Dombrovski, A. Y., Parker, A. M., \& Szanto, K. (2016). Late-life depression, suicidal ideation, and attempted suicide: The role of individual differences in maximizing, regret, and negative decision outcomes. Journal of Personality and Social Psychology, 29 (4), 363-371.

Burnside, W. (1971). Judgment of short time intervals while performing mathematical tasks. Perception \& Psychophysics, 9, 404-406.

Carmeci, F., Misuraca, R., \& Cardaci, M. (2009). A study of temporal estimation from the perspective of the mental clock model. The Journal of General Psychology, 136(2), 117-128.

Cheek, N. N., \& Schwartz, B. (2016). On the meaning and measurement of maximization. Judgment and Decision Making, 11(2), 126-146.

Chernev, A. (2003). When more is less and less is more: The role of ideal point availability and assortment in consumer choice. Journal of Consumer Research, 30(2), 170-183.

Chowdhury, T. G., Ratneshwar, S., \& Mohanty, P. (2009). The time-harried shopper: Exploring the differences between maximizers and satisficers. Marketing Letters, 20(2), 155-167.

Coelho, M., Ferreira, J. J., Dias, B., Sampaio, C., Martins, I. P., \& CastroCaldas, A. (2004). Assessment of time perception: The effect of aging. Journal of the International Neuropsychological Society, 10(03), 332-341.

Dalal, D. K., Diab, D. L., Zhu, X., \& Hwang, T. (2015). Understanding the construct of maximizing tendency: A theoretical and empirical evaluation. Journal of Behavioral Decision Making, 28, 437-450.

Dar-Nimrod, I., Rawn, C. D., Lehman, D. R., \& Schwartz, B. (2009). The maximization paradox: The costs of seeking alternatives. Personality and Individual Differences, 46(5), 631-635. 
De Palma, A., Myers, G. M., \& Papageorgiou, Y. Y. (1994). Rational choice under an imperfect ability to choose. The American Economic Review, 419-440.

DeShazo, J. R., \& Fermo, G. (2002). Designing choice sets for stated preference methods: The effects of complexity on choice consistency. Journal of Environmental Economics and Management, 44(1), 123-143.

Diab, D. L., Gillespie, M. A., \& Highhouse, S. (2008). Are maximizers really unhappy? The measurement of maximizing tendency. Judgment and Decision Making, 3(5), 364-370.

Dijksterhuis, A., Bos, M. W., Nordgren, L. F., \& Van Baaren, R. B. (2006). On making the right choice: The deliberation-without-attention effect. Science, 311(5763), 1005-1007.

Fasolo, B., Carmeci, F. A., \& Misuraca, R. (2009). The effect of choice complexity on perception of time spent choosing: When choice takes longer but feels shorter. Psychology \& Marketing, 26, 213-228.

Fasolo, B., Hertwig, R., Huber, M., \& Ludwig, M. (2009). Size, entropy, and density: What is the difference that makes the difference between small and large real-world assortments? Psychology \& Marketing, 26(3), 254-279.

Fasolo, B., McClelland, G. H., \& Todd, P. M. (2007). Escaping the tyranny of choice: When fewer attributes make choice easier. Marketing Theory, 7, 13-26.

Haynes, G. A. (2009). Testing the boundaries of the choice overload phenomenon: The effect of number of options and time pressure on decision difficulty and satisfaction. Psychology \& Marketing, 26, 204-212.

Hox, J. (2010). Multilevel modelling: Techniques and applications (2nd ed.). New York, NY: Routledge.

lyengar, S. S., \& Kamenica, E. (2010). Choice proliferation, simplicity seeking, and asset allocation. Journal of Public Economics, 94(7), 530-539.

lyengar, S. S., \& Lepper, M. R. (2000). When choice is demotivating: Can one desire too much of a good thing? Journal of Personality and Social Psychology, 79(6), 995-1006.

lyengar, S. S., Wells, R. E., \& Schwartz, B. (2006). Doing better but feeling worse looking for the "best" job undermines satisfaction. Psychological Science, 17(2), 143-150.

Jessup, R. K., Veinott, E. S., Todd, P. M., \& Busemeyer, J. R. (2009). Leaving the store empty-handed: Testing explanations for the too-muchchoice effect using decision field theory. Psychology \& Marketing, 26 (3), 299-320.

Kahneman, D. (1973). Attention and effort. Englewood Cliffs, NJ: Prentice-Hall.

Kahneman, D., \& Snell, J. (1992). Predicting a changing taste: Do people know what they will like? Journal of Behavioral Decision Making, 5(3), 187-200.

Keller, K. L., \& Staelin, R. (1987). Effects of quality and quantity of information on decision effectiveness. Journal of Consumer Research, 200-213.

Kokkoris, M. D. (2016). Revisiting the relationship between maximizing and well-being: An investigation of eudaimonic well-being. Personality and Individual Differences, 99, 174-178.

Mantel, S. P., \& Kellaris, J. J. (2003). Cognitive determinants of consumers' time perceptions: The impact of resources required and available. Journal of Consumer Research, 29(4), 531-538.

Misuraca, R., Faraci, P., Gangemi, A., Carmeci, F. A., \& Miceli, S. (2015). The decision making tendency inventory: A new measure to assess maximizing, satisficing, and minimizing. Personality and Individual Differences, 85, 111-116.

Misuraca, R., \& Teuscher, U. (2013). Time flies when you maximizeMaximizers and satisficers perceive time differently when making decisions. Acta Psychologica, 143, 176-180.

Nenkov, G. Y., Morrin, M., Ward, A., Schwartz, B., \& Hulland, J. (2008). A short form of the maximization scale: Factor structure, reliability and validity studies. Judgment and Decision Making, 3(5), 371-388.
Pande, B., \& Pati, A. K. (2010). Overestimation/underestimation of time: Concept confusion hoodwink conclusion. Biological Rhythm Research, 41(5), 379-390.

Parker, A. M., Bruine de Bruin, W., \& Fischhoff, B. (2007). Maximizers versus satisficers: Decision-making styles, competence, and outcomes. Judgment and Decision Making, 2(6), 342-350.

Purvis, A., Howell, R. T., \& lyer, R. (2011). Exploring the role of personality in the relationship between maximization and well-being. Personality and Individual Differences, 50, 370-375.

Rogge, N. (2016). Love is blind: How our love for more choice costs time. Psychology \& Marketing, 33(5), 358-371.

Ryan, R. M., \& Deci, E. L. (2000). Self-determination theory and the facilitation of intrinsic motivation, social development, and well-being. American Psychologist, 55(1), 68-78.

Sanchis, R. G., Rey, J. M., \& Álvarez, F. (2014). Numerical analysis of a time allocation model accounting for choice overload. International Journal of Computer Mathematics, 91(2), 315-326.

Scheibehenne, B., Greifeneder, R., \& Todd, P. M. (2010). Can there ever be too many options? A meta-analytic review of choice overload. Journal of Consumer Research, 37(3), 409-425.

Schwartz, B. (2004). The paradox of choice-Why more is less. New York, NY: Harper Collins.

Schwartz, B., Ward, A., Monterosso, J., Lyubomirsky, S., White, K., \& Lehman, D. R. (2002). Maximizing versus satisficing: Happiness is a matter of choice. Journal of Personality and Social Psychology, 83(5), 1178.

Shah, A. M., \& Wolford, G. (2007). Buying behavior as a function of parametric variation of number of choices. Psychological Science, 18(5), 369-370.

Simon, H. A. (1955). A behavioral model of rational choice. The Quarterly Journal of Economics, 69, 99-118.

Simon, H. A. (1956). Rational choice and structure of the environment. Psychological Review, 63, 129-138.

Snibbe, A. C., \& Markus, H. R. (2005). You can't always get what you want: Educational attainment, agency, and choice. Journal of Personality and Social Psychology, 88(4), 703-720.

Sparks, E. A., Ehrlinger, J., \& Eibach, R. P. (2012). Failing to commit: Maximizers avoid commitment in a way that contributes to reduced satisfaction. Personality and Individual Differences, 52(1), 72-77.

Thomas, E. A. C., \& Weaver, W. B. (1975). Cognitive processing and time perception. Perception \& Psychophysics, 17, 363-367.

Turner, B. M., Rim, H. B., Betz, N. E., \& Nygren, T. E. (2012). The maximization inventory. Judgment and Decision Making, 7(1), 48-60.

Von Neumann, J., \& Morgenstern, O. (1953). Theory of games and economic behaviour (3rd ed.). Princeton, NJ: Princeton University Press.

Wearden, J. H. (2005). The wrong tree: Time perception and time experience in the elderly. In J. Duncan, L. Phillips, \& P. McLeod (Eds.), Measuring the mind: Speed, age, and control (pp. 137-158). Oxford, UK: Oxford University Press.

White, C. M., \& Hoffrage, U. (2009). Testing the tyranny of too much choice against the allure of more choice. Psychology \& Marketing, 26 (3), 280-298.

Woehrle, J. L., \& Magliano, J. P. (2012). Time flies faster if a person has a high working-memory capacity. Acta Psychologica, 139(2), 314-319.

Zakay, D., \& Block, R. A. (1997). Temporal cognition. Current Directions in Psychological Science, 6, 12-18.

Zakay, D., \& Block, R. A. (2004). Prospective and retrospective duration judgments: An executive-control perspective. Acta Neurobiologiae Experimentalis, 64(3), 319-328.

Nicky Rogge is a Professor of Economics at the Faculty of Business and Economics of the KULeuven (Belgium). He has three key research areas: (a) the construction of composite indicators for policy evaluation using the benefit-of-the-doubt methodology 
and related techniques, (b) efficiency analysis of organizations and entities (both private and public) in different operation or policy environments using (non)parametric efficiency measurement techniques, and (c) (economic) analysis of decision and choice-making behavior of economic agents. In all three research areas, he has published articles in international peer-reviewed academic journals.

How to cite this article: Rogge N. Maximizing, choice freedom, and duration judgments in choice making. J Consumer Behav. 2017;1-14. https://doi.org/10.1002/cb.1668

\section{Appendix}

\section{EXAMPLE OF CHOICE TASK ABOUT SMARTPHONES}

A. | Suppose that your smartphone broke down due to a nasty fall and that you are currently looking for a new one. In your search for a smartphone, you consult a Web shop for smartphones that is known for its good service and state-of-the-art smartphone collection. Below is a selection of five smartphones as suggested by this Web shop based on some search queries. The Web shop provides information on seven important specifications. Rank the five smartphones in order of preference starting with the most preferred smartphone.

\begin{tabular}{|c|c|c|c|c|c|}
\hline Specifications & Smartphone 1 & Smartphone 2 & Smartphone 3 & Smartphone 4 & Smartphone 5 \\
\hline Screen size (in.) & 4 & 4.8 & 4 & 4.7 & 2.44 \\
\hline Price $(€)$ & 252.95 & 398.95 & 196.95 & 611.78 & 169.99 \\
\hline Quality operating system (score on 10) & 9 & 10 & 7 & 10 & 9 \\
\hline Weight (g) & 120 & 143 & 114 & 103 & 93 \\
\hline
\end{tabular}

